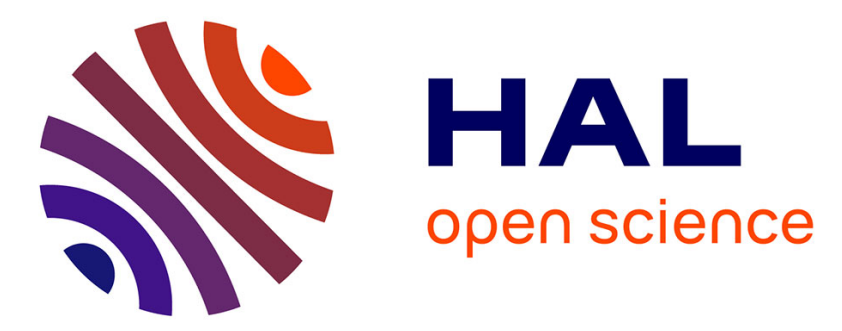

\title{
Hysteresis During Stress-Induced Variant Rearrangement
}

\author{
J. Ball, C. Chu, R. James
}

\section{To cite this version:}

J. Ball, C. Chu, R. James. Hysteresis During Stress-Induced Variant Rearrangement. Journal de Physique IV Proceedings, 1995, 05 (C8), pp.C8-245-C8-251. 10.1051/jp4:1995834 . jpa-00254083

\section{HAL Id: jpa-00254083 https://hal.science/jpa-00254083}

Submitted on 1 Jan 1995

HAL is a multi-disciplinary open access archive for the deposit and dissemination of scientific research documents, whether they are published or not. The documents may come from teaching and research institutions in France or abroad, or from public or private research centers.
L'archive ouverte pluridisciplinaire HAL, est destinée au dépôt et à la diffusion de documents scientifiques de niveau recherche, publiés ou non, émanant des établissements d'enseignement et de recherche français ou étrangers, des laboratoires publics ou privés. 


\title{
Hysteresis During Stress-Induced Variant Rearrangement
}

\author{
J.M. Ball, C. Chu* and R.D. James* \\ Department of Mathematics, Heriot-Watt University, Riccarton, Edinburgh EH14 4AS, Scotland \\ * Department of Aerospace Engineering and Mechanics, 107 Akerman Hall, 110 Union Street S.E., \\ University of Minnesota, Minneapolis, MN 55455, U.S.A.
}

\begin{abstract}
This paper represents an attempt to understand, from basic principles, the origins of hysteresis during variant rearrangement. As a framework for this study, we focus on the biaxial loading experiments conducted in [7] on single crystals of $\gamma_{1}^{\prime}$ martensite, oriented so that two compound twinned variants of martensite have least energy. Our analysis supports the idea that hysteresis (at least in these slow cyclic experiments) is due to metastability: as the loads are changed, the current state goes from stable to metastable to unstable. The idea we explore is that the metastability is essentially caused by geometric incompatibility. That is, even though there is a state of lower energy than the metastable state, it is necessarily geometrically incompatible with it, and this gives rise to an energy barrier. We show that this concept of metastability (based on calculating relative minimizers of energy) has a close relation with the Schmid Law and reveals an interesting dependence on the shape of the specimen. Further details and background for the ideas presented here can be found in [4], forthcoming.
\end{abstract}

\section{BIAXIAL LOADING EXPERIMENTS}

To test any idea of metastability as it relates to hysteresis, it is helpful to have a set of experiments for which the beginning and ending states are single-variant (so that one need only assess the metastability of of the single variant state) and for which there are several independent control parameters, so that the idea is rigorously tested. The experiments of $\mathrm{Chu}$ and James $[1,7,8]$ are of this type. They are orthogonal biaxial loading experiments on single crystals of $\mathrm{Cu}-14.0 \mathrm{wt} \% \mathrm{Al}-3.9 \mathrm{wt}$. $\% \mathrm{Ni}$ in the fully transformed $\gamma_{1}^{\prime}$ martensitic phase. The loading device in these experiments was specifically designed to be governed by the free energy,

$$
-\int_{\Omega} \mathbf{T} \cdot \nabla \mathbf{y}(\mathbf{x}) d \mathbf{x}
$$

where $\mathbf{y}(\mathbf{x})$ is the deformation (defined on the reference configuration $\Omega$ ) and $\mathbf{T}$ is a constant $3 \times 3$ matrix of the form

$$
\mathbf{T}=\left(\begin{array}{ccc}
\sigma_{1} & 0 & 0 \\
0 & \sigma_{2} & 0 \\
0 & 0 & 0
\end{array}\right)
$$

in a certain fixed basis $\mathbf{e}_{1}, \mathbf{e}_{2}, \mathbf{e}_{3}$ (the "machine basis"). The two constants $\sigma_{1}$ and $\sigma_{2}$ are the engineering stresses applied to the edges of the specimen. See [4] for further background and [7] for the technical details of how the design of a machine based on (1) was carried out; briefiy, the loading wires are attached to the edge of the specimen (a thin square plate $2.54 \mathrm{~cm} \times 2.54 \mathrm{~cm}$ with normal (100)) and all loading wires along one edge pull with approximately the same force, no matter how the specimen deforms. The resultant forces in the two directions $\mathbf{e}_{1}, \mathbf{e}_{2}$ can be independently controlled and, by varying the ratio of these forces, the stable variant can be made to shift. It was noted in these experiments that the technical details of constructing a loading device faithful to (1) are important; if slight changes in the loading device are made, then the hysteresis loops change considerably, even when the evolution of the resultant forces remains the same.

The reason for designing a loading device that accurately simulates the energy (1) is that, if the total free energy of the specimen and loading device has the form $([2,3])$,

$$
\int_{\Omega} \varphi(\nabla \mathbf{y}(\mathbf{x}), \theta) d \mathbf{x}-\int_{\Omega} \mathbf{T} \cdot \nabla \mathbf{y}(\mathbf{x}) d \mathbf{x}
$$

i.e., the energy of the specimen is given by the first integral in (3), then (under mild hypotheses on $\varphi$ ) linear deformations minimize energy. That is, if the $3 \times 3$ matrix $\mathbf{G}=\mathbf{A}$ minimizes the integrand $\varphi(\mathbf{G}, \theta)-\mathbf{T} \cdot \mathbf{G}$, 
then the deformation $\mathbf{y}(\mathbf{x})=$ Ax minimizes the total energy (3) relative to all other deformations. In (3), $\theta$ is the temperature.

In this machine specimens of essentially any orientation can be meaningfully tested, i.e. the specimen axes do not have to line up with the machine axes. For the tests considered here the specimen was oriented so that, according to the calculation presented in Section 2 below, just two of the six possible variants of martensite have lowest energy for all values of the loads, and these two variants are compound twinned. These particular variants were chosen because it is known from [10] that the twin interfaces in this case are extremely mobile, and for this study we wanted to explore metastability alone.

The results of the tests of interest here are as follows ([7]). For most values of $\sigma_{1}$ and $\sigma_{2}$ the specimen was observed to be homogeneously deformed in a single variant of martensite (say, variant 2), as expected based on the design of the machine. As the loads changed, this deformation changed slightly due to elastic deformation of this variant. Occasionally, on certain loading paths, this deformation became unstable at certain values of the stresses $\sigma_{1}^{\text {crit. }}$ and $\sigma_{2}^{\text {crit. }}$, and there occurred a rapid avalanche of variant rearrangement, involving microstructures of the two variants, that led to a single, homogeneously deformation of variant 1. Continuing on this loading path, the deformation remained homogeneously deformed in variant 1 . Reversing the loading path, the specimen remained in variant 1 until certain critical loads, different from $\sigma_{1}^{\text {crit. }}$ and $\sigma_{2}^{\text {crit. }}$, were reached, and there followed an abrupt transition back to variant 2. A plot of the volume fraction $\lambda$ of variant 1 as a function of $\sigma_{1}-\sigma_{2}$, on a loading program in which $\sigma_{1}+\sigma_{2}$ was held constant, is shown in Figure 1.

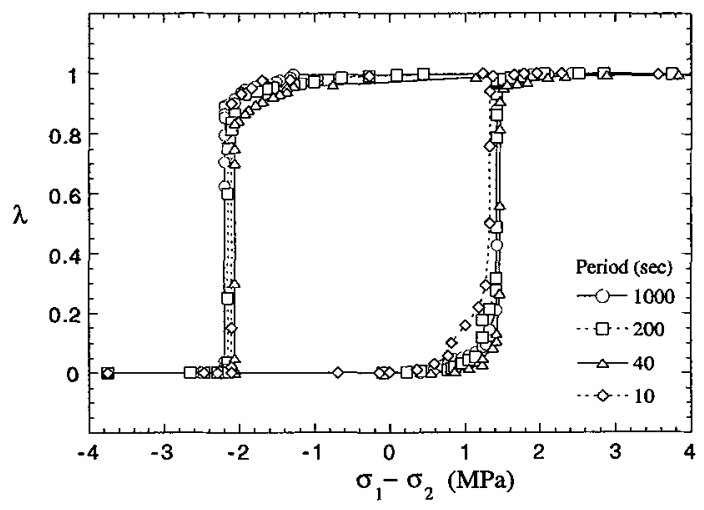

Figure 1. Typical outer loop: $\lambda$ vs. $\sigma_{1}-\sigma_{2}$ with $\sigma_{1}+\sigma_{2}=10.7 \mathrm{MPa}$.

Note the squareness of the hysteresis loop and the absence of a rate-effect at these rates. From these loops the transition stresses can be identified as the values corresponding to $\lambda=0.5$. Of course, these values of depend on the loading path, and it was found that for various loading paths these values corresponding to the variant $1 \rightarrow 2$ transition fall nearly on a straight line, and those corresponding to the variant $2 \rightarrow 1$ transition fall on a parallel line, as shown in Figure 2. 


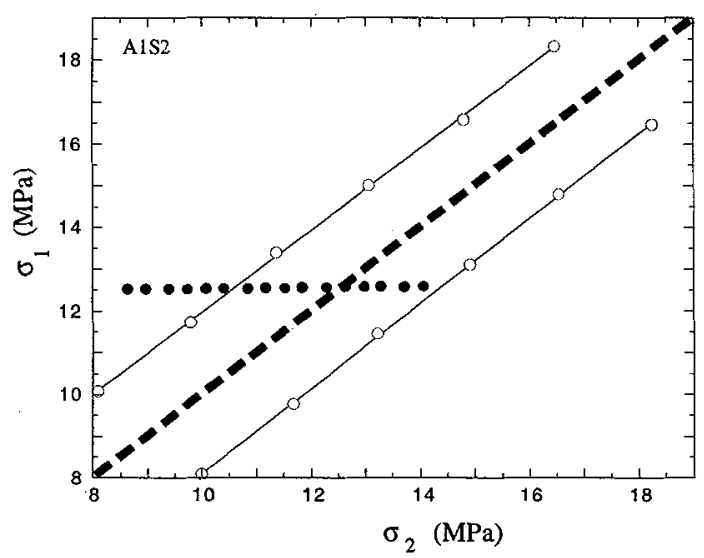

Figure 2. Effect of loading path. The circles are experimental, the dashed line is given by the minimum energy calculation of Section 2 and the dotted line is the loading path described in Section 3.

The distance between these two lines is a measure of the size of the hysteresis. In incremental tests, with a relatively long waiting period between increments, it was found that transition occurred also on two straight lines a little inside of the solid lines pictured in Figure 2, as might be guessed from the slight roundedness of the shoulders of the hysteresis loop shown in Figure 1. In fact, the transition stresses were found to be sensitive to imposed disturbances: by tapping lightly on the loading wires, the transition could be induced at stresses somewhat inside the lines shown in Figure 2. Thus, the identification of the transition stresses as the values corresponding to $\lambda=0.5$ is a little arbitrary, and the actual stresses at transition depend on the disturbances present, which originally motivated us to consider metastability as the source of hysteresis.

Specimen orientation had a significant effect on hysteresis, as shown in Figure 3. All specimens used in the tests shown in Figure 3 had face normal (100) (cubic basis) but had edge normals (110), (1-10) for A1S2, $(0.34,0.76,0.56),(0.047,0.58,-0.82)$ for I-10, $(0.34,0.88,0.35),(-0.043,0.36,-0.93)$ for I-9 and $(0.21,0.88$, $-0.43),(-0.27,-0.37,-0.89)$ for I-6.

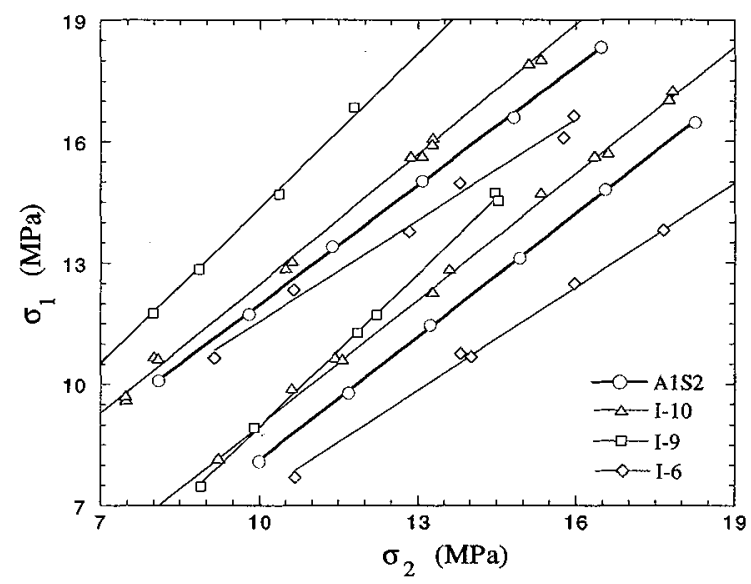

Figure 3. Effect of orientation on hysteresis. See text. 


\section{MINIMUM ENERGY}

For the $\gamma_{1}^{\prime}$ martensite studied here, $\varphi(\mathbf{G}, \theta)$ has six energy wells corresponding to the six variants of martensite $([2,5])$. These are defined by the six matrices,

$$
\begin{array}{ll}
\mathbf{U}_{1}=\left(\begin{array}{ccc}
\frac{\alpha+\gamma}{2} & \frac{\alpha-\gamma}{2} & 0 \\
\frac{\alpha-\gamma}{2} & \frac{\alpha+\gamma}{2} & 0 \\
0 & 0 & \beta
\end{array}\right), & \mathbf{U}_{2}=\left(\begin{array}{ccc}
\frac{\alpha+\gamma}{2} & \frac{\gamma-\alpha}{2} & 0 \\
\frac{\gamma-\alpha}{2} & \frac{\alpha+\gamma}{2} & 0 \\
0 & 0 & \beta
\end{array}\right), \\
\mathbf{U}_{3}=\left(\begin{array}{ccc}
\frac{\alpha+\gamma}{2} & 0 & \frac{\alpha-\gamma}{2} \\
0 & \beta & 0 \\
\frac{\alpha-\gamma}{2} & 0 & \frac{\alpha+\gamma}{2}
\end{array}\right), & \mathbf{U}_{4}=\left(\begin{array}{ccc}
\frac{\alpha+\gamma}{2} & 0 & \frac{\gamma-\alpha}{2} \\
0 & \beta & 0 \\
\frac{\gamma-\alpha}{2} & 0 & \frac{\alpha+\gamma}{2}
\end{array}\right), \\
\mathbf{U}_{5}=\left(\begin{array}{ccc}
\beta & 0 & 0 \\
0 & \frac{\alpha+\gamma}{2} & \frac{\alpha-\gamma}{2} \\
0 & \frac{\alpha-\gamma}{2} & \frac{\alpha+\gamma}{2}
\end{array}\right), & \mathbf{U}_{6}=\left(\begin{array}{ccc}
\beta & 0 & 0 \\
0 & \frac{\alpha+\gamma}{2} & \frac{\gamma-\alpha}{2} \\
0 & \frac{\gamma-\alpha}{2} & \frac{\alpha+\gamma}{2}
\end{array}\right),
\end{array}
$$

in the cubic basis, where $\alpha=1.0619, \beta=0.9178$ and $\gamma=1.0230$. That is, suppressing the dependence of $\varphi$ on $\theta$ (since the experiments described above were done at a fixed temperature below $A_{s}$ ) we assume that $\varphi$ has minima at these matrices. Since by general principles $\varphi$ must also be frame-indifferent, $\varphi(\mathbf{R G})=\varphi(\mathbf{G})$ holds for all $3 \times 3$ matrices $\mathbf{R}$ in the set $S O(3)=\left\{\mathbf{R}: \mathbf{R}^{T} \mathbf{R}=\mathbf{1}\right.$, $\left.\operatorname{det} \mathbf{R}=\mathbf{1}\right\}$ and all $3 \times 3$ matrices $\mathbf{G}$, then $\varphi$ must also be minimized at all matrices of the form $\mathbf{R U}_{1}, \ldots, \mathbf{R} \mathbf{U}_{6}$, where $\mathbf{R} \in S O(3)$. For simplicity, we let $M=S O(3) \mathbf{U}_{1} \cup \ldots \cup S O(3) \mathbf{U}_{6}$ be the collection of all the energy wells.

As discussed above, the problem

$$
\min _{\mathbf{G}}\{\varphi(\mathbf{G})-\mathbf{T} \cdot \mathbf{G}\}
$$

serves to determine energy minimizing homogeneous deformations. One can do a direct calculation of the minimizing deformation gradients from (5), after using the measured linear elastic moduli of the material to determine the shape of $\varphi$ near each of its energy wells. Because of the fact that all the stresses used in the experiments were much smaller than these linear elastic moduli, the minimizers of (5) lie exceedingly close to the energy wells and, one obtains an excellent approximation to these minimizers by minimizing (5) under the additional constraint $\mathbf{G} \in M$ (see [4] for further discussion). Throughout the rest of this paper we shall operate within this constrained theory, for simplicity. This simplifies (5), since $\varphi=$ const. on $M$, and really reflects the fact that the loading device energy dominates the specimen energy [1]. With this constraint and the properties of $\varphi$ we have assumed, (5) becomes

$$
\min _{i \in\{i, \ldots, 6\}, \mathbf{R} \in S O(3)}\left\{\text { const. }-\mathbf{T} \cdot \mathbf{R U}_{i}\right\}
$$

The solution of (6) is a straightforward algebraic calculation [4,7]. The result depends strongly on orientation, i.e. the relation between the machine basis and the cubic basis, as expected based on the form of (6). For all of the orientations represented in Figure 3 and for $\sigma_{1}>0$ and $\sigma_{2}>0$, the minimum in (6) is attained with $i$ $=1$ or 2 . Having said this, the best way to express the result is to draw the locus of points in the $\sigma_{1}, \sigma_{2}$ plane at which variants 1 and 2 have the same minimum energy, i.e., at which (6) has equi- minimizers with $i=1$ or 2 . This locus is shown as the dashed line in Figure 2 for that orientation. Below this line variant 2 is stable; above this line variant 1 is stable. It is seen that the dashed line nearly bisects the two lines that define the size of the hysteresis, and this also occurs for the other orientations tested (Note that in Figure 3, the slopes of the pairs of transition lines are different for different orientations). However, a minimum energy calculation cannot explain the size of the hysteresis.

\section{METASTABILITY AND GEOMETRIC INCOMPATIBILITY}

Before explaining the notion of metastability we believe is operative in these experiments, we recall the 
conditions of compatibility. A deformation $\mathbf{y}(\mathbf{x})$ of the form

$$
\mathbf{y}(\mathbf{x})= \begin{cases}\mathbf{A x}, & \mathbf{x} \cdot \mathbf{n} \leq 0 \\ \mathbf{B x}, & \mathbf{x} \cdot \mathbf{n}>0, \quad \mathbf{A} \neq \mathbf{B},\end{cases}
$$

is continuous ("fiducial scratches can bend sharply but never break") if and only if $\operatorname{rank}(\mathbf{B}-\mathbf{A})=1$. We use the terminology "A and $\mathbf{B}$ are rank-1 connected" if $\operatorname{rank}(\mathbf{B}-\mathbf{A})=1$. The pairs of rank-1 connected matrices that lie on distinct energy wells describe the possible twinning deformations in the material [2]. For example, if $\mathbf{A}=\mathbf{R U _ { 1 }}$ for some rotation matrix $\mathbf{R}$, then there are precisely two matrices, $\mathbf{B}_{1}=\mathbf{R}_{1} \mathbf{U}_{2}$ and $\mathbf{B}_{2}=\mathbf{R}_{2} \mathbf{U}_{2}$, on $S O(3) \mathrm{U}_{2}$ that are rank-1 connected to $\mathrm{A}$, and these describe the reciprocal twins. Explicit formulas for the rotations $\mathbf{R}_{1}$ and $\mathbf{R}_{2}$ are given in [4].

To be definite, consider a loading path $\sigma_{1}=$ const. $>0$ and $\sigma_{2}$ increasing from zero. If $\sigma_{2}>0$ is small, variant 1 is stable (according to the minimum energy calculation (6)), and a homogeneous deformation closely given by $\mathbf{y}(\mathbf{x})=\mathbf{R U}_{1} \mathbf{x}, \mathbf{R} \in S O(3)$, is observed in the experiments (It was difficult comparing the predicted value of the rotation matrix $\mathbf{R}$ based on (6) with observations, because of the difficulty of establishing a reference for measuring angles). For each value of $\sigma_{2}>0$ there is a unique rotation matrix $\mathbf{R}\left(\sigma_{2}\right)$ that minimizes $-\mathbf{T} \cdot \mathbf{R} \mathbf{U}_{1}$. The deformation $\mathbf{y}(\mathbf{x})=\mathbf{R}\left(\sigma_{2}\right) \mathbf{U}_{1} \mathbf{x}$ is the absolute minimizer of the energy until $\left(\sigma_{1}, \sigma_{2}\right)$ reaches the dashed line of Figure 2. Now continue increasing $\sigma_{2}$ so that $\left(\sigma_{1}, \sigma_{2}\right)$ is a little past the dashed line. Then variant 1 no longer minimizes the energy, and there is a rotation matrix $\widehat{\mathbf{R}}\left(\sigma_{2}\right)$ such that $\mathbf{y}(\mathbf{x})=\widehat{\mathbf{R}}\left(\sigma_{2}\right) \mathbf{U}_{2} \mathbf{x}$ has minimum energy. In fact, there is a small set $S$ of matrices containing $\widehat{\mathbf{R}}\left(\sigma_{2}\right) \mathbf{U}_{2}$ such that the associated deformations have less energy than $\mathbf{y}(\mathbf{x})=\mathbf{R}\left(\sigma_{2}\right) \mathbf{U}_{1} \mathbf{x}$. One can calculate explicitly these matrices and one finds by direct calculation that $\mathbf{R}\left(\sigma_{2}\right) \mathbf{U}_{1}$ is not rank-1 connected to any of them. In summary, we are at a pair $\left(\sigma_{1}, \sigma_{2}\right)$ a little to the right of the dashed line in Figure 2, we are considering the metastablility of the deformation $\mathbf{y}(\mathbf{x})=\mathbf{R}\left(\sigma_{2}\right) \mathbf{U}_{1} \mathbf{x}$, there is a small set $S$ of $3 \times 3$ matrices on the other well $S O(3) \mathbf{U}_{2}$ that have a smaller value of the energy density than $\mathbf{R}\left(\sigma_{2}\right) \mathbf{U}_{1}$, and none of the matrices in $S$ is rank-1 connected to $\mathbf{R}\left(\sigma_{2}\right) \mathbf{U}_{1}$.

Now suppose we consider a small disturbance of the deformation $\mathbf{y}(\mathbf{x})=\mathbf{R}\left(\sigma_{2}\right) \mathbf{U}_{1} \mathbf{x}$. By "small" we have in mind that the deformation of the disturbed state remains close to $\mathbf{y}(\mathbf{x})=\mathbf{R}\left(\sigma_{2}\right) \mathbf{U}_{1} \mathbf{x}$, but that its deformation gradient may be far from $\mathbf{R}\left(\sigma_{2}\right) \mathbf{U}_{1}$ (To keep the deformation of the disturbed state close to $\mathbf{y}(\mathbf{x})=\mathbf{R}\left(\sigma_{2}\right) \mathbf{U}_{1} \mathbf{x}$, it follows necessarily that its deformation gradient can only stray far from $\mathbf{R}\left(\sigma_{2}\right) \mathbf{U}_{1}$ on a small part of the specimen). Thus, the problem of metastability is to show that all such small disturbances have more energy than the given state. This is quite plausible, in that there are no rank-1 connections between $S$ and $\mathbf{R}\left(\sigma_{2}\right) \mathbf{U}_{1}$. Indeed it is shown in [4] that all such sufficiently small disturbances have more energy than $\mathbf{y}(\mathbf{x})=\mathbf{R}\left(\sigma_{2}\right) \mathbf{U}_{1} \mathbf{x}$ (See [4] for the precise statement). The idea behind the argument is the following: any small region with deformation gradient $\nabla \mathbf{y} \in S$ necessarily must be surrounded by a transition layer on which $\nabla \mathbf{y}$ is far from both wells, due to the lack of rank-1 connections, and one "pays" more in energy for the transition layer than one gains by having $\nabla \mathbf{y} \in S$ (see [11] for an earlier version of this kind of argument). The argument is tricky because the lack of rank-1 connections between deformation gradients does not in itself prevent microstructures being constructed from them (see [6]) but, in this case, because all the deformation gradients in question are near just two matrices that are not rank-1 connected, such microstuctures are ruled out. The argument does not adopt a particular "shape" of the disturbance, and there is a version of it that applies when interfacial energy contributions are also included.

Unfortunately, our methods are not yet sufficiently refined to say precisely where metastability is lost, but only say that if $\left(\sigma_{1}, \sigma_{2}\right)$ is a little to the right of the dashed line, the state $\mathbf{y}(\mathbf{x})=\mathbf{R}\left(\sigma_{2}\right) \mathbf{U}_{1} \mathbf{x}$ remains metastable.

\section{THE SCHMID LAW AND THE EFFECT OF SHAPE}

While we are not yet able to predict a precise "size of the hysteresis," it is easy to get an upper bound. Again, for definitness, we consider the loading path described above with $\sigma_{1}$ fixed. As we increase $\sigma_{2}$ to the right of the dashed line in Figure 2, the set $S$ of matrices (with energy density less than $\left.\mathbf{R}\left(\sigma_{2}\right) \mathbf{U}_{1}\right)$ grows. Eventually, we reach a first stress $\sigma_{2}=\sigma_{2}^{*}$ such that for $\sigma_{2}>\sigma_{2}^{*} S$ contains a matrix that is rank-1 connected to $\mathbf{R}\left(\sigma_{2}^{*}\right) \mathbf{U}_{1}$ (Recall that $\mathbf{R}\left(\sigma_{2}^{*}\right) \mathbf{U}_{1}$ is rank-1 connected to exactly 2 matrices on the well $\left.S O(3) \mathbf{U}_{2}\right)$. Let $\mathbf{B}^{*}$ be rank-1 connected to $\mathbf{R}\left(\sigma_{2}\right) \mathbf{U}_{1}$ for $\sigma_{2}$ slightly greater than $\sigma_{2}^{*}, \mathbf{B}^{*}-\mathbf{R}\left(\sigma_{2}\right) \mathbf{U}_{1}=\mathbf{a} \otimes \mathbf{n}$. Now make the following elementary construction shown in Figure 4: a deformation with a thin twin band with normal $\mathbf{n}$ and having the deformation gradient B*. $^{*}$. 


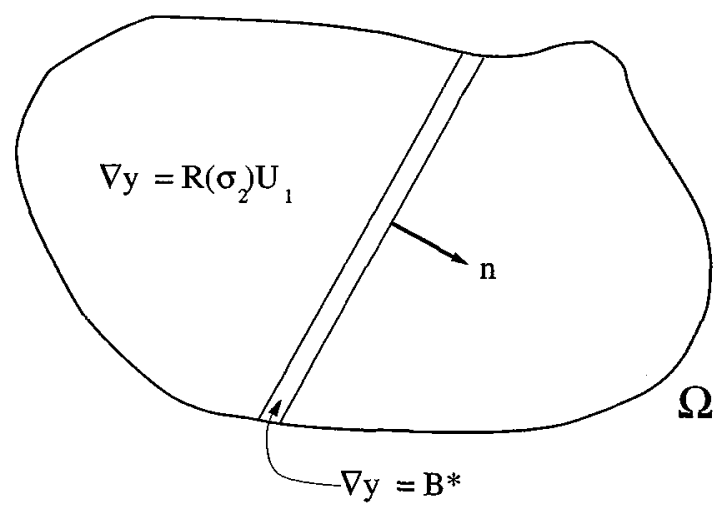

Figure 4. A deformation that yields an upper bound to the size of the hysteresis when $\sigma_{2}>\sigma_{2}^{*}$.

By construction, this deformation can be made as close to $\mathbf{y}(\mathbf{x})=\mathbf{R}\left(\sigma_{2}\right) \mathbf{U}_{1} \mathbf{x}$ as desired (by making the twin band thin), it lowers the energy, and it is compatible. Hence, $\mathbf{y}(\mathbf{x})=\mathbf{R}\left(\sigma_{2}\right) \mathbf{U}_{1} \mathbf{x}$ fails to be metastable for $\sigma_{2}>\sigma_{2}^{*}$. Combining the conditions that $\mathbf{B}^{*}$ be rank-1 connected to $\mathbf{R}\left(\sigma_{2}\right) \mathbf{U}_{1}$ and that $\mathbf{B}^{*}$ have a smaller energy density than $\mathbf{R}\left(\sigma_{2}\right) \mathbf{U}_{1}$, we have

$$
0<\mathbf{T} \cdot \mathbf{B}^{*}-\mathbf{T} \cdot \mathbf{R}\left(\sigma_{2}\right) \mathbf{U}_{1}=\mathbf{a} \cdot \mathbf{T n}
$$

But this last inequality is just the Schmid Law (with Schmid constant 0). This establishes a direct relation between the Schmid law and metastability, which we believe represents the physical basis of this law for variant rearrangement. The condition that defines $\sigma_{2}^{*}$, i.e., $\mathbf{a} \cdot \mathbf{T n}=0$, can also be plotted for various orientations using forward and reverse loading paths. This compares reasonably well with the experimental values of the size of the hysteresis based on the incremental tests, but underestimates the size of the hysteresis shown above in Figure 3 (See [4] for a detailed comparison). If we also include interfacial energy, our essential argument would remain unchanged: a construction like Figure 4 would also give a Schmid law, but with a positive constant. Note that with finite deformations considered, as in the present study, a Schmid constant of 0 still gives hysteresis.

The Schmid Law gives an upper bound for metastability, but could it be a sharp upper bound? In general, the answer is no within the present framework. If the specimen has an appropriate sharp corner, then, surprizingly, more complicated deformations lower the energy before $\sigma_{2}^{*}$ is reached. The details can be

found in [4], and are framed in the context of a slight generalization of the constrained theory which allows for transition layers. The argument relies on the compatible microstructure pictured in Figure 5. The idea is that the deformation gradient $\mathbf{C}$ is "well-inside" the region $S$ and strongly lowers the energy but need not be rank-1 connected to $\mathbf{R}\left(\sigma_{2}\right) \mathbf{U}_{1}$ because of the intermediary laminate.

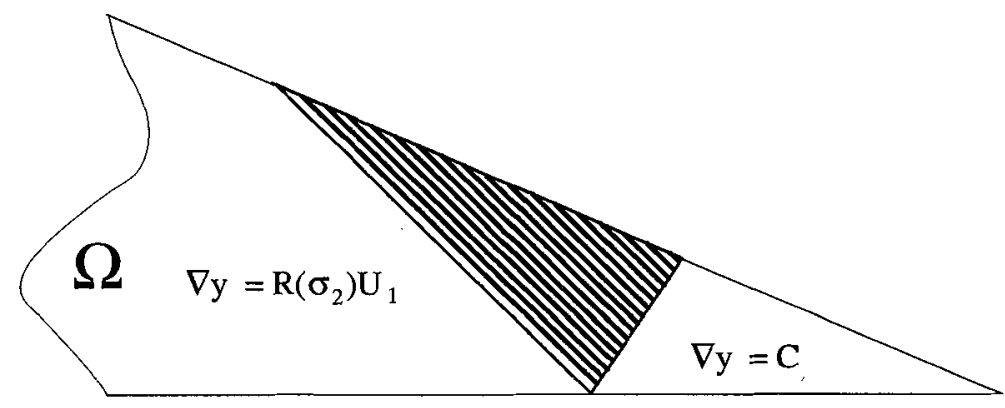

Figure 5. Microstructure that lowers the energy at stresses below the Schmid stresses for a pointed specimen.

This argument, which needs further study, suggests that one might lower the hysteresis by placing on the specimen certain outward pointing sharp corners. Paradoxically, in ferromagnetism, certain inward pointing 
corners drastically reduce the $\mathbf{M}$ vs. $\mathbf{H}$ hysteresis; however, this is apparently closely related to the behavior of the magnetic field near such corners.

\section{Acknowledgement}

R.D. James thanks ONR (N00014-93-1-0240, N00014-91-J-4034), AFOSR (AFOSR-91-0301) and NSF (CMS-95-03633, DMS-9111572-02) and J.M. Ball thanks EU (ERBSCI** CT000670) and EPSRC (GR/J03466) for supporting this work.

\section{References}

[1] Abeyaratne R., Chu C. and James R.D., submitted to Phil. Mag.

[2] Ball J.M. and James R.D., Phil. Trans. Royal Soc. London A 338 1992), pp. 389-450.

[3] Ball J.M. and James R.D., International Conference on Martensitic Transformation, July 20-24, 1993, C.M. Wayman and J. Perkins Eds. (Monterey Institute for Advanced Studies, 1993), pp. 65-76.

[4] Ball J.M., Chu C. and James R.D., preprint.

[5] Bhattacharya K., Acta. Met. 219 (1991), pp. 2431-2444.

[6] Bhattacharya K., Kohn R., James R.D. and Firoozye N., Proc. Roy. Soc. Edinburgh 124A (1994), pp. 843-878.

[7] Chu C., Thesis, University of Minnesota (1993).

[8] Chu C. and James R.D., Experiments in Smart Materials and Structures, ASME, AMD-Vol. 181 (1993).

[9] Chu C. and James R.D., ICOMAT-95, to appear.

[10] Ichinoise S., Funatsu Y. and Otsuka K., Acta Metall. et Materialia 33 (1985), pp. 1613-1620.

[11] Kohn R.V. and Sternberg P., Proc. Roy. Soc. Edinburgh 111A (1989), pp 69-84. 